\title{
Yellow Pattern 577-nm Micropulse Laser: Treatment of Macular Edema from Radiation Retinopathy - A Case Report
}

\author{
James G. Wong ${ }^{a-c} \quad$ Trang T.H. Nguyen ${ }^{a}$ \\ ${ }^{a}$ Strathfield Retina Clinic, Sydney, NSW, Australia; ${ }^{b}$ Medical Retina Unit, \\ Sydney Eye Hospital, Sydney, NSW, Australia; 'Save Sight Institute, University of \\ Sydney, Sydney, NSW, Australia
}

\section{Keywords}

Micropulse $\cdot$ Laser $\cdot$ Subthreshold $\cdot$ Radiation retinopathy $\cdot$ Macular edema

\begin{abstract}
We report a case of a 60-year-old Asian male who developed radiation retinopathy 23 years after initial radiotherapy for nasopharyngeal carcinoma and was successfully treated with yellow pattern 577-nm micropulse laser. Secondary macular edema and visual acuity improved following a single treatment session with minimal scarring. Yellow pattern micropulse laser is a safe and effective treatment for macular edema secondary to radiation retinopathy.

(C) 2017 The Author(s)

Published by S. Karger AG, Basel
\end{abstract}

\section{Introduction}

Radiation retinopathy is a chronic and progressive retinal microangiopathy that can occur even years after radiation treatment for cancer. Radiation retinopathy has been reported secondary to treatment of intraocular tumors, such as uveal melanoma, retinoblastoma, as well as cephalic, nasopharyngeal, orbital, and paranasal tumors [1-3]. Pathological vascular changes include a pattern of capillary occlusion, dilatation, and leakage, often within the 
macula which may progress to microaneurysms, telangiectatic-like vessels exudation and subsequent cystoid macular edema (CME) [4]. Capillary nonperfusion may also result in vasoproliferative sequelae, including retinal neovascularization, secondary vitreous hemorrhage, and neovascular glaucoma. Current treatment of radiation retinopathy includes thermal laser photocoagulation, intravitreal antivascular endothelial growth factor (anti-VEGF) injections, intravitreal steroid injections, and hyperbaric oxygen [5]. However, despite treatment patients often may have progressive visual loss due to the significant amount of ischemic damage. CME secondary to radiation retinopathy may often be challenging to treat.

Conventional thermal laser photocoagulation for CME may result in macular scarring and eventually vision loss due to progressive atrophy from direct laser scars. Subthreshold micropulse laser has been increasingly recognized as an effective and safe treatment for CME secondary to other retinal vascular diseases, including diabetic macular edema, retinal vein occlusion, and central serous retinopathy [6]. Unlike conventional thermal laser, micropulse laser delivers short bursts of laser energy in microsecond pulses - micropulses - rather than a continuous wave, resulting in less thermal damage and minimal or no scarring. Also, yellow $(577 \mathrm{~nm})$ wavelength laser light may be theoretically safer than conventional green $(532 \mathrm{~nm})$ wavelength laser light when treating the macular area due to less thermal absorption by the xanthophyll pigments [6]. Here, we report a case of CME secondary to radiation retinopathy which was treated successfully with yellow pattern $577-\mathrm{nm}$ micropulse laser.

\section{Case Report}

A 60-year-old Asian man presented with a 2-month history of reduced vision and metamorphopsia in the left eye. He had mild controlled hypertension and hypercholesterolemia. $\mathrm{He}$ also had a history of nasopharyngeal carcinoma which was treated with local radiation and chemotherapy 23 years and 9 years previously.

Best-corrected visual acuity (BCVA) was 20/16 in the right eye and 20/40 in the left eye. Fundoscopy showed some intraretinal hemorrhages in the left temporal macula with telangiectatic vessels and scattered microaneurysms (Fig. 1). Fluorescein angiography revealed patchy capillaries, nonperfusion, and left late macular leak consistent with CME (Fig. 2). Optical coherence tomography (OCT; Heidelberg Engineering, Heidelberg, Germany) showed left CME (Fig. 3a). Following informed consent, a solid-state subthreshold yellow 577-nm wavelength pattern micropulse laser (Supra Scan 577; Quantel Medical, Cournon d'Auvergne, France) with an adapter attached to a Haag-Streit type slit-lamp microscope was used to treat the left eye. Using fluorescein angiography guidance to treat the leaking areas, single-spot and 9-spot square patterns of $100-\mu \mathrm{m}$ diameter were used with a duration of $0.02 \mathrm{~s}$ and a duty cycle of $15 \%$ (the laser stayed on for $15 \%$ of the time).

Two months following treatment, subjectively the patient noted improved symptoms, BCVA had improved to 20/20 in the left eye and the OCT examination demonstrated reduced intraretinal edema (Fig. 3b). Ten months after the micropulse laser, BCVA had stabilized to $20 / 20$ in the left eye and the OCT examination revealed absence of the previously found intraretinal edema (Fig. 3c). 


\section{Case Reports in Ophthalmology}

\section{Discussion}

Subthreshold micropulse laser is an innovative therapy for retinal disorders which has been shown to be safe and effective in the treatment of macular edema [6, 7]. The yellow pattern 577-nm micropulse laser in this case converts each laser shot into a "pulse envelope" consisting of a sequence of extremely short pulses of laser (10-20 ms) with intervening periods of zero laser energy to the treatment site. When the multi-spot pattern mode is used, the pulses are not produced simultaneously; however, they formed in rapid succession with low-energy pulses during each laser shot. Laser generated in this way is typically $10-25 \%$ of the visible threshold power. Previous studies have shown that subthreshold laser performed in this way was sufficient to show a consistent retinal pigment epithelium-confined photothermal effect, and also to allow the inner retinal temperature to remain below the threshold of coagulative damage, sparing the transparency of the retina and lowering the risk of hemorrhage [6]. Because the micropulse laser treatment has no or minimal damage to the retina or choroid, and is less likely to produce chorioretinal scars, retreatment of the same area is feasible. As a result, it is possible to safely treat macular edema in a transfoveal location using subthreshold micropulse laser. However, the possibility of undertreatment is always a concern due to the difficulty in titrating treatment without a visible end point [8]. In our case, we utilized fluorescein angiography guidance to treat the leaking areas (overlay of the treatment map provided in Fig. 2). A previous study demonstrated that indocyanine green dye-guided micropulse diode laser was effective in the treatment of central serous chorioretinopathy [9].

The pathogenesis of radiation retinopathy still remains elusive. In 1994, in a review, Archer et al. [4] proposed that initial radiation caused the immediate death of retinal endovascular cells which created microscopic foci of epithelial damage. A vicious cycle of retinal vascular endothelial cell death, migration, division, and further death thus ensues, outstretching the ability of endothelial cells to maintain an unbroken endothelial lining and triggering inflammation and clotting cascades $[4,5]$. As a result, occlusion of discrete retinal capillary beds creates hemodynamic stress on neighboring capillaries which dilate to accommodate [4]. However, many such capillary collaterals are compromised by radiation damage, leading to vascular leakage or further occlusion [4]. Occurring within the macular area, such vascular leakage results in CME. Our patient presented with retinopathy and macular edema 23 years after initial radiotherapy. The onset of pathological changes may possibly be delayed due to the slow turnover of retinal vascular endothelial cells. Our patient with macular edema secondary to radiation retinopathy was successfully treated with 1 session of subthreshold yellow pattern 577-nm micropulse laser with no detectable complications or retinal scarring. The molecular mechanisms underlying treatment success of micropulse laser are poorly understood. Postulated processes include modification of inflammatory cytokines or induction of heat shock proteins in retinal pigment epithelium cells [10].

In this case report, we presented a 60-year-old Asian man with radiation retinopathy who was treated successfully with 1 session of yellow pattern 577-nm micropulse laser photocoagulation, resulting in improving symptoms and fluid resolution. Micropulse laser photocoagulation therapy is an effective treatment for CME secondary to radiation retinopathy. Further studies are needed to elucidate possible mechanisms of subthreshold laser and to develop better therapeutic as well as preventive treatment strategies for radiation retinopathy. 


\section{Case Reports in Ophthalmology} www.karger.com/cop

Wong and Nguyen: Yellow Pattern 577-nm Micropulse Laser: Treatment of Macula Edema from Radiation Retinopathy - A Case Report

\section{Statement of Ethics}

The patient was informed that imaging related to his case would not be able to identify him personally and may be published and he consented for the publication.

\section{Disclosure Statement}

The authors declare that no conflicts of interest are involved in the present case report.

\section{References}

-1 Egbert PR, Donaldson SS, Moazed K, Rosenthal AR: Visual results and ocular complications following radiotherapy for retinoblastoma. Arch Ophthalmol 1978;96:1826-1830.

Amoaku WM, Archer DB: Cephalic radiation and retinal vasculopathy. Eye 1990;4:195-203.

3 Bianciotto C, Shields CL, Pirondini C, Mashayekhi A, Furuta M, Shields JA: Proliferative radiation retinopathy after plaque radiotherapy for uveal melanoma. Ophthalmology 2010;117:1005-1012. Archer DB, Gardiner TA: Ionizing radiation and the retina. Curr Opin Ophthalmol 1994;5:59-65. Giuliari GP, Sadaka A, Hinkle DM, Simpson ER: Current treatments for radiation retinopathy. Acta Oncol 2011;50:6-13.

-6 Vujosevic S, Martini F, Longhin E, Convento E, Cavarzeran F, Midena E: Subthreshold micropulse yellow laser versus subthreshold micropulse infrared laser in center-involving diabetic macular rdema: morphologic and functional safety. Retina 2015;35:1594-1603.

7 Othman IS, Eissa SA, Kotb MS, Sadek SH: Subthreshold diode-laser micropulse photocoagulation as a primary and secondary line of treatment in management of diabetic macular edema. Clin Ophthalmol 2014;8:653-659.

8 Sivaprasad S, Elagouz M, McHugh D, Shona O, Dorin G: Micropulsed diode laser therapy: evolution and clinical applications. Surv Ophthalmol 2010;55:516-530.

-9 Ricci F, Missiroli F, Regine F, Grossi M, Dorin G: Indocyanine green enhanced subthreshold diode-laser micropulse photocoagulation treatment of chronic central serous chorioretinopathy. Graefes Arch Clin Exp Ophthalmol 2009;247:597-607.

10 Inagaki K, Shuo T, Katakura K, Ebihara N, Murakami A, Ohkoshi K: Sublethal photothermal dtimulation with a micropulse laser induces heat shock protein rxpression in ARPE-19 vells. J Ophthalmol 2015;2015:729792.
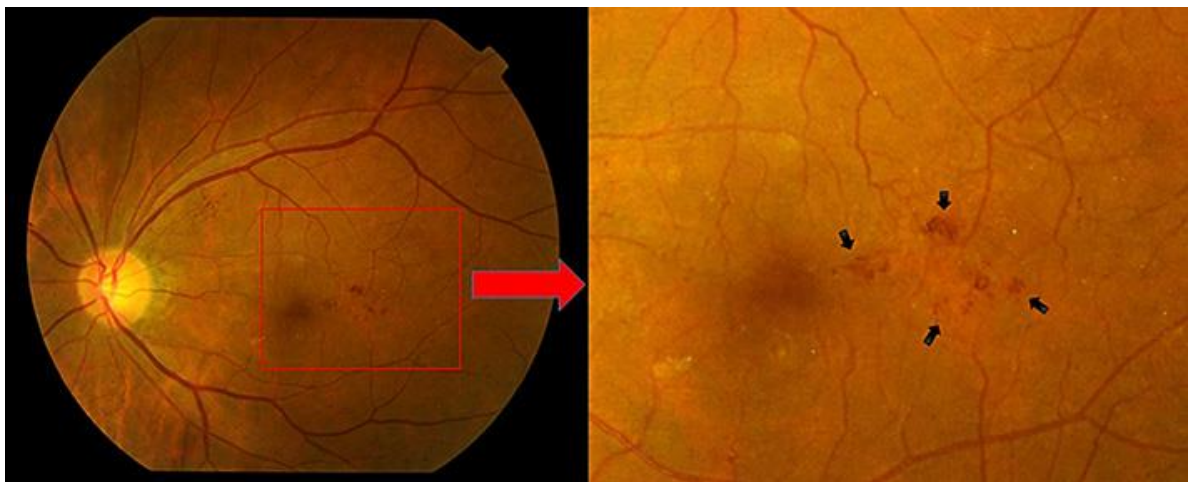

Fig. 1. Fundoscopy showing the left macular area with intraretinal hemorrhages, telangiectatic vessels, and scattered microaneurysms before micropulse laser treatment. 


\section{Case Reports in Ophthalmology} Case Rep Ophthalmol 2017;8:81-86 DOI: 10.1159/000456028

(c) 2017 The Author(s). Published by S. Karger AG, Basel www.karger.com/cop

Wong and Nguyen: Yellow Pattern 577-nm Micropulse Laser: Treatment of Macular Edema from Radiation Retinopathy - A Case Report

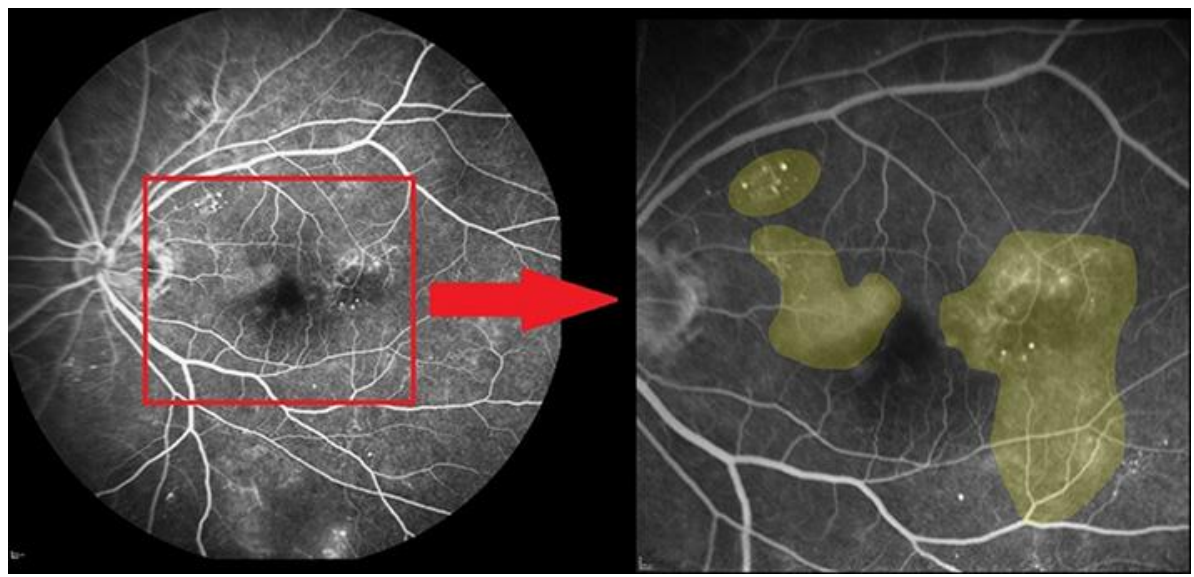

Fig. 2. Fluorescein angiography demonstrating radiation retinopathy with microangiopathic findings of patchy capillaries, nonperfusion, and late leak in the left macula before laser treatment. An overlay (yellow-shaded area) showing the fluorescein-guided treatment area. 


\section{Case Reports in Ophthalmology} Case Rep Ophthalmol 2017;8:81-86 DOI: 10.1159/000456028 (c) www.karger.com/cop

Wong and Nguyen: Yellow Pattern 577-nm Micropulse Laser: Treatment of Macular Edema from Radiation Retinopathy - A Case Report
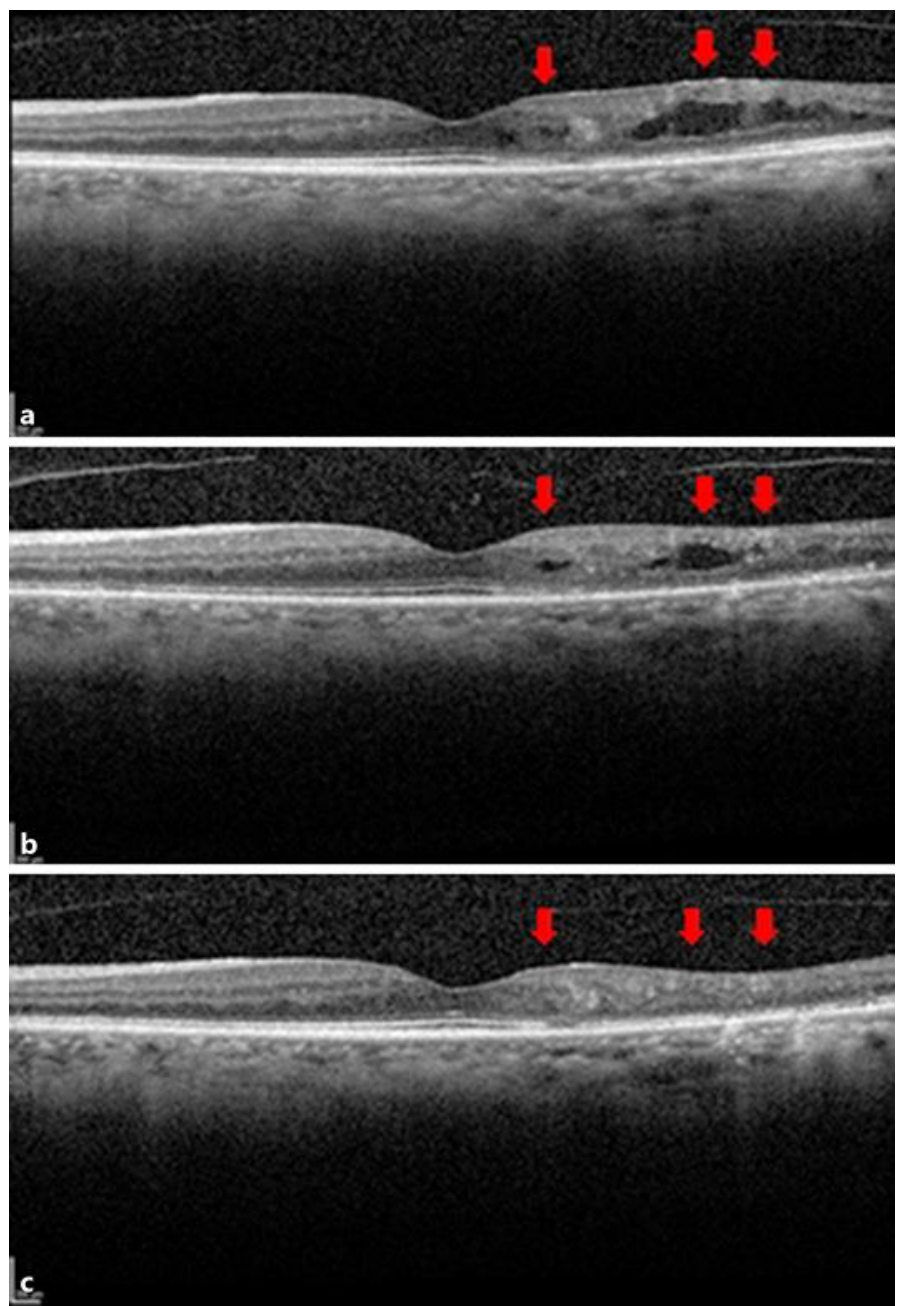

Fig. 3. OCT showing left CME (arrows) secondary to radiation retinopathy at baseline (a). Reduction of intraretinal edema 2 months after laser (b). Improvement of the macular edema at 10 months after micropulse laser therapy $(\mathbf{c})$. 